\title{
MOLECULAR AND FUNCTIONAL CHARACTERIZATION OF
} AUTOPHAGY MARKER LC3 IN ORANGE-SPOTTED GROUPER (EPINEPHELUS COIOIDES)

\author{
Chao-Fen Lin ${ }^{1,2}$, Chee-Shin Chua ${ }^{1,3}$, Young-Mao Chen ${ }^{1,3,4}$, Tzong-Yueh Chen ${ }^{1,2,3,4}$ § \\ ${ }^{1}$ Institute of Biotechnology, National Cheng Kung University, Tainan 70101, Taiwan \\ ${ }^{2}$ Department of Biotechnology and Bioindustry Sciences, National Cheng Kung University, Tainan \\ 70101, Taiwan \\ ${ }^{3}$ Translational Center for Marine Biotechnology, National Cheng Kung University, Tainan 70101, \\ Taiwan
}

${ }^{4}$ Agriculture Biotechnology Research Center, National Cheng Kung University, Tainan 70101, Taiwan

\begin{abstract}
Autophagy is a conserved cellular process in response to stress, sustain normal cell growth by degrade and recycle unnecessary cytosolic components in autophagosome and autolysosome. Microtubuleassociated protein light chain 3 known as LC3 is a major constituent of the autophagosome and used as a reliable marker for autophagy. In this study, a full length of orange-spotted grouper LC3 (osgLC3) mRNA had been cloned with a size of 1,440 bp including the sequence encoded for a 126 a.a. protein. The phylogenetic relationship and relative tissue mRNA expression levels for osgLC3 has been evaluated. The punctate structures increased in the GF-1 cells with over-expressed osgLC 3 under nutrient deprivation revealed that starvation induced autophagy formation and the colocalization of punctate structures and lysosome indicated autolysosome formation during autophagy induction. Additionally, it has found that autophagy formation in GF-1 cells induced by nervous necrosis virus (NNV) infection through quantitation of endogenous osgLC3-positive punctate structures. In grouper larvae, the mRNA expression of osgLC3 were increased under NNV infection and the osgLC3 was able to convert to lipidated form of LC3 (osgLC3-II) for synthesis of autophagosome. Moreover, it has been verified that Hsp90 plays a role in autophagy and here the osgLC3 and Hsp90AB had higher FRET efficiency that implied the interaction between osgLC3 and Hsp90AB. Based on these results, osgLC3 contributed to the autophagy formation in response to NNV infection in grouper and the working mechanisms of osgLC3 and Hsp90AB during virus infection need further studies.
\end{abstract}

\section{KEYWORDS}

LC3, Autophagy, Epinephelus coioides, Autophagosome, Nervous necrosis virus

§Corresponding author. Tel.: +886-6-2757575\#65622-610; Fax: +886-6-2766505

E-mail address: ibcty@mail.ncku.edu.tw 